\title{
Fault Diagnosis of Nonlinear Uncertain Systems with Triangular Form
}

\author{
Qi Ding, Xiafu Peng, Xunyu Zhong, and Xiaoqiang Hu \\ Department of Automation, Xiamen University, Xiamen 361005, China \\ Correspondence should be addressed to Xiafu Peng; pengxiafu@sina.cn
}

Received 31 March 2017; Revised 14 June 2017; Accepted 28 June 2017; Published 1 August 2017

Academic Editor: Chunhui Zhao

Copyright (C) 2017 Qi Ding et al. This is an open access article distributed under the Creative Commons Attribution License, which permits unrestricted use, distribution, and reproduction in any medium, provided the original work is properly cited.

\begin{abstract}
A novel approach to fault diagnosis for a class of nonlinear uncertain systems with triangular form is proposed in this paper. It is based on the extended state observer (ESO) of the active disturbance rejection controller and linearization of dynamic compensation. Firstly, an ESO is designed to jointly estimate the states and the combination of uncertainty, faults, and nonlinear function of nonlinear uncertain systems. It can derive the estimation of nonlinear function via the state estimations and system model. Then, linearization of dynamic compensation is employed to linearize the system by offsetting nonlinear function mandatorily using its estimation. An observer-based residual generator is designed on the basis of the prior linearized model for fault diagnosis. Moreover, threshold treatment technique is adopted to improve the robustness of fault diagnosis. This method is utilizable and simple in construction and parameter tuning. And also we show the construction of ESO and give the corresponding convergence proof succinctly. Finally, a numerical example is presented to illustrate the validity of the proposed fault diagnosis scheme.
\end{abstract}

\section{Introduction}

In the past few decades, fault diagnosis technology has been advanced by leaps and bounds, and there has been significant research activity in the design and analysis of fault diagnosis [1-4]. Furthermore, the method based on analytic models is one of the most pioneer and in-depth approaches in fault diagnosis area (see, e.g., [5-14]). Model-based diagnosis can make great use of the system information and has a high diagnosis precision for fault location, fault type judgement, and amplitude estimation of fault. It is mainly applicable for linear systems, whereas in many practical situations, nonlinear properties of the control systems cannot be ignored in dealing with fault diagnosis problem. Therefore, it has practical significance in research fault diagnosis method for nonlinear systems based on analytic models [15].

At present, model-based fault diagnosis for nonlinear systems is generally based upon two kinds of basic theory: One way is to linearize the original nonlinear system near its working point using state feedback linearization technique to obtain the linear model. And the residual is designed based on unknown input decoupled technique to minimize the influence of the modeling error which is treated as unknown input. The other is that nonlinear observer method or nonlinear parameter estimation method is applied to fault diagnosis for nonlinear systems directly, without resort to any linearization. In a word, model-based fault diagnosis can be defined as the determination of faults of a system from the comparison of available system measurements with a prior information represented by the system's mathematical model through generation of residual quantities and their analysis [16]. A back-propagation neural network fault detection method was employed in [17] to detect the proton exchange membrane fuel cell (PEMFC) current fault type. According to the diagnosis results, the reconfiguration mechanism determined three nonlinear controllers based on feedback linearization approaches which are, respectively, built to adjust the voltage and pressure difference in the case of normal to be selected. The sliding mode observer for the nonlinear fault diagnosis was designed to observe the whole nonlinear system and actuator in [18]. Then the faults are diagnosed according to the residual value changes of two observers. In [15], the system model was decomposed by $(B, K, \phi)$ and the observer with high sensitivity for faults and strong robustness for unknown input disturbances was designed by using the decoupling technology. Reference [19] 
proposed a new approach based on adaptive sliding mode observer to detect, isolate, and identify faults to deal with the shortcomings that existing model-based fault diagnosis methods of aircraft engines require high precision engine model. Reference [20] established a time-varying failure model of aeroengine control and designed an adaptive observer to estimate the state, actuator, and sensor faults of the system simultaneously. In recent years, various diagnosis methods based on the knowledge have been proposed, including neural network method, rough set-based method, genetic algorithm-based method, expert system method, and wavelet transform method. But all these methods have welldefined control limits [21].

The novel method broke through some limitations in this paper, compared with the above-mentioned methods, as assumption of measurable system variables and amplitude and type limitation about faults. It can be concluded from theoretical analysis and simulation results that the proposed method is easily used to identify the fault and get its approximate function, which differs from traditional methods. Reference [22] investigated fault diagnosis method based on ESO which estimated several faults directly. References [23, 24] also estimated fault directly using ESO to get its approximate function. Without doubt, this method took uncertainty as part of the fault in uncertain systems, which led to large error of estimated fault and actual fault. Reference [25] used the same technique, but here too there was the same issue about misinformation and inaccurate fault estimation. In short, this method has poor robustness.

In this paper, system variables are estimated to derive the estimation of nonlinear function using ESO with high tracking accuracy, high estimation precision, and less sensitivity to model errors and external disturbances. Then, linearization of dynamic compensation is employed to linearize the system via estimated value. And the residual system is constructed based on full-order state observer for the prior linearized model to get approximate function of fault. Moreover, in order to strengthen the robustness of fault diagnosis, this paper adopts threshold value treatment technique. Eventually the complex fault diagnosis problem of nonlinear system is simplified as one of linear system to perform the task of fault diagnosis, and the method is robust.

The rest of this paper is organized as follows. In Section 2, the problem of nonlinear fault diagnosis is formulated. The proposed fault diagnosis scheme is presented in Section 3. In Section 4, the derivation of threshold for robust fault detection is given. Section 5 describes a simulation example to illustrate the effectiveness of the robust fault diagnosis method. Finally, Section 6 presents some concluding remarks.

\section{Problem Statement}

Consider a class of uncertain nonlinear systems described by

$$
\begin{aligned}
\dot{\bar{x}}(t) & =\psi(\bar{x}(t), \omega(t))+B u(t)+B_{f} f(t), \\
y(t) & =C \bar{x}(t),
\end{aligned}
$$

where $\bar{x}(t) \in R^{n}, u(t) \in R$, and $y(t) \in R$ are the system state vector, input vector, and output vector, respectively. $f(t) \in R$ is the dynamic process fault of nonlinear system, and $\omega(t) \in R$ is the disturbance or uncertainty of the system. $\psi: R^{n} \rightarrow R^{n}$ is a real nonlinear vector function and has the form as follows:

$$
\psi(\bar{x}(t), \omega(t))=\left[\begin{array}{c}
\psi_{1}\left(\bar{x}_{1}, \bar{x}_{2}\right) \\
\vdots \\
\psi_{i}\left(\bar{x}_{1}, \ldots, \bar{x}_{i+1}\right) \\
\vdots \\
\psi_{n-1}\left(\bar{x}_{1}, \ldots, \bar{x}_{n-1}\right)+\bar{x}_{n} \\
\psi_{n}\left(\bar{x}_{1}, \ldots, \bar{x}_{n}\right)
\end{array}\right] .
$$

$B=[0,0,0, \ldots, b]_{1 \times n}^{T}$ is the input matrix. $C=[1,0,0, \ldots$, $0]_{1 \times n}$ is the output matrix. In addition, $B_{f}=[0,0,0, \ldots, 1]_{1 \times n}^{T}$ is the fault coefficient matrix.

There are many practical applications of such systems, such as Vander Pol oscillator system and robot arm system. Research on fault diagnosis for such systems has both theoretical and practical importance. It is shown in [26] that the coordinate change

$$
\begin{aligned}
& x_{1}=\bar{x}_{1}, \\
& \dot{x}_{i}=x_{i+1}, \quad i=1,2, \ldots, n-1, \\
& \dot{x}_{n}=\phi\left(x_{1}, x_{2}, \ldots, x_{n}, t\right)+\omega(t)+b u(t)+f(t) .
\end{aligned}
$$

transforms the system into the triangular form

$$
\begin{aligned}
\dot{x}_{i} & =x_{i+1}, \quad i=1,2, \ldots, n-1, \\
\dot{x}_{n} & =\phi\left(x_{1}, x_{2}, \ldots, x_{n}, t\right)+\omega(t)+b u(t)+f(t), \\
y & =x_{1},
\end{aligned}
$$

where $\phi$ represents nonlinear function of the transformed system (4).

2.1. System Variables Estimation. In this paper, we estimate the system variables by designing ESO with high tracking accuracy and high estimation precision.

By defining a new system state in system (4) as $x_{n+1}=$ $\phi\left(x_{1}, x_{2}, \ldots, x_{n}, t\right)+\omega(t)+f(t)$, the augmented system can be obtained as

$$
\begin{aligned}
\dot{x}_{i} & =x_{i+1}, \quad i=1,2, \ldots, n-1, \\
\dot{x}_{n} & =x_{n+1}+b u_{0}(t), \\
\dot{x}_{n+1} & =\dot{\phi}+\dot{\omega}+\dot{f}=\varphi(x, t), \\
y & =x_{1} .
\end{aligned}
$$

In order to track the system states $x_{1}, \ldots, x_{n}$ efficiently, in this work it is proposed to extend uncertainty, faults, and 
nonlinear function as a new system state $x_{n+1}$ and design an ESO as follows:

$$
\begin{gathered}
\tilde{x}_{1}=z_{1}(t)-y(t), \\
\dot{z}_{i}=z_{i+1}(t)-\beta_{i} g_{i}\left(\tilde{x}_{1}\right), \quad i=1,2, \ldots, n-1, \\
\vdots \\
\dot{z}_{n}=z_{n+1}(t)-\beta_{n} g_{n}\left(\tilde{x}_{1}\right)+b u(t), \\
\dot{z}_{n+1}=-\beta_{n+1} g_{n+1}\left(\tilde{x}_{1}\right),
\end{gathered}
$$

where $z(t) \in R^{n+1}$ and $u(t) \in R$ are ESO state and input. $\beta_{i}(i=1, \ldots, n+1)$ are observer parameters, and $\beta_{1}=$ $1 / h, \ldots, \beta_{i}=\left(0.2+10^{2-i}\right) / h^{i}(i=2, \ldots, n+1)$. Based on the experience, the observer parameter can be roughly selected and adjusted in the light of Fibonacci sequence. $h$ is sampling period. Also the function $g\left(\tilde{x}_{1}\right)$ can be expressed as

$$
g_{i}\left(\tilde{x}_{1}\right)= \begin{cases}\left|\tilde{x}_{1}\right|^{a} \operatorname{sign}\left(\tilde{x}_{1}\right), & \left|\tilde{x}_{1}\right|>\delta \\ \frac{\tilde{x}_{1}}{\delta^{1-a}}, & \left|\tilde{x}_{1}\right| \leq \delta, \\ & (i=1,2, \ldots, n+1),\end{cases}
$$

where $a \in(0,1)$ and $\delta$ is range length of linear interval which is of a minor amount.

Let $\tilde{x}_{i}=x_{i}-z_{i}, i=1,2, \ldots, n+1$. From (5) and (6), the observer estimation error can be shown as

$$
\begin{gathered}
\dot{\tilde{x}}_{1}=\tilde{x}_{2}+\beta_{1} g_{1}\left(\tilde{x}_{1}\right), \\
\vdots \\
\dot{\tilde{x}}_{n-1}=\tilde{x}_{n}+\beta_{n-1} g_{n-1}\left(\tilde{x}_{1}\right), \\
\dot{\tilde{x}}_{n}=\tilde{x}_{n+1}+\beta_{n} g_{n}\left(\tilde{x}_{1}\right), \\
\dot{\tilde{x}}_{n+1}=\varphi(x, t)+\beta_{n+1} g_{n+1}\left(\tilde{x}_{1}\right) .
\end{gathered}
$$

Theorem 1. Denoting the Jacobian matrix of system (8) as $A_{1}(\tilde{x})$, let $F(\tilde{x})=\dot{\tilde{x}} ;$ that is, $A_{1}(\tilde{x})=\partial F / \partial \tilde{x}$. If there exist two symmetric positive definite matrices $P_{1}, Q_{1}$, such that $\forall \tilde{x} \neq 0$, the matrix $S(\tilde{x})=A_{1}^{T} P_{1}+P_{1} A_{1}+Q_{1}$ is negative semidefinite in some neighborhood $\Omega$ of the origin. Then system (8) is asymptotically stable.

Proof. For the symmetric positive definite matrices $P_{1}, Q_{1}$, let us consider a Lyapunov function candidate

$$
V_{1}(\tilde{x})=F^{T} P_{1} F
$$

Assuming that $A_{1}(\tilde{x})$ is singular, then one can find a nonzero vector $y_{0}$ such that $A_{1}(\tilde{x}) y_{0}=0$. Since

$$
y_{0}^{T} S y_{0}=y_{0}^{T} A_{1}^{T} P_{1} y_{0}+y_{0}^{T} P_{1} A_{1} y_{0}+y_{0}^{T} Q_{1} y_{0}
$$

the singularity of $A_{1}$ implies that $y_{0}^{T} A_{1}^{T} P_{1} y_{0}+y_{0}^{T} P_{1} A_{1} y_{0}=0$; we can get

$$
y_{0}^{T} S y_{0}=y_{0}^{T} Q_{1} y_{0}
$$

which contradicts the assumed negative definiteness of $S$.
The invertibility and continuity of $A_{1}$ guarantee that the function $F(\tilde{x})$ can be uniquely inverted. This implies that $F(\tilde{x}) \neq 0$ for $\tilde{x} \neq 0$. Given the above result, the function $V_{1}(\tilde{x})=F^{T} P_{1} F$ is positive definite.

The derivative of $V_{1}$ can be written

$$
\dot{V}_{1}(\tilde{x})=\dot{F}^{T} P_{1} F+F^{T} P_{1} \dot{F} .
$$

Using the fact that $\dot{F}=A_{1} F$, we can get

$$
\dot{V}_{1}(\tilde{x})=F^{T} A_{1}^{T} P_{1} F+F^{T} P_{1} A_{1} \dot{F}=F^{T} S F-F^{T} Q_{1} F .
$$

Because $S$ is negative semidefinite and $Q_{1}$ is positive definite, $\dot{V}_{1}$ is negative definite. If $V_{1}(\tilde{x}) \rightarrow \infty$ as $\|\tilde{x}\| \rightarrow \infty$, system (8) is globally asymptotically stable based on Lyapunov theorem.

Note that as long as we select appropriately parameters $\beta_{1}, \ldots, \beta_{n+1}$, ESO can estimate both states $x_{1}(t), \ldots, x_{n}(t)$ of system (4) and the extended state $x_{n+1}(t)=\phi+\omega+f$ very well; that is,

$$
\begin{gathered}
z_{1}(t) \longrightarrow x_{1}(t), \ldots, z_{n}(t) \longrightarrow x_{n}(t), \\
z_{n+1}(t) \longrightarrow x_{n+1}(t)=\phi\left(x_{1}, x_{2}, \ldots, x_{n}, t\right)+\omega(t)+f(t) .
\end{gathered}
$$

Also ESO is evolved by the optimal control, and large quantities engineering experiences from practice show that ESO is convergent.

2.2. Linearization Process. The estimation of nonlinear function $\widehat{\phi}\left(z_{1}, z_{2}, \ldots, z_{n}, t\right)$ can be derived and the extended state estimation $z_{n+1}(t)$ can be got with $n$ system state estimations and system model. On this basis we substitute the estimation of nonlinear function for its value and then select appropriately control variable to achieve linearization of dynamic compensation. In this work, the control variable is given by

$$
u(t)=u_{0}-\frac{\widehat{\phi}}{b}
$$

then nonlinear system (4) is turned into linear integrator series control system (16); that is,

$$
\begin{aligned}
& \dot{x}_{i}=x_{i+1}(t), \quad i=1,2, \ldots, n-1, \\
& \dot{x}_{n}=b u_{0}(t)+\omega(t)+f(t), \\
& y=x_{1},
\end{aligned}
$$

where $\omega(t)$ is the sum of not eliminated uncertainty and estimation errors.

It is easy to know that system state estimations contain disturbing information to make uncertainty weakened by linearization of dynamic compensation.

\section{Fault Diagnosis}

As mentioned above, nonlinear system (4) is turned into linear integrator series control system (16) by linearization of dynamic compensation which simplifies fault diagnosis of nonlinear system into that of linear system, whereafter a fullorder fault detection filter is designed for linearized system, 
achieving convergence of observation error as well as fault diagnosis based on residual error system. Linear integrator series control system (16) can be uniformly expressed in state equations of general forms as, distinctly,

$$
\begin{aligned}
& \dot{x}=A x(t)+B u_{0}(t)+B_{\omega} \omega(t)+B_{f} f(t), \\
& y=C x,
\end{aligned}
$$

with

$$
\begin{aligned}
& A=\left[\begin{array}{ccccc}
0 & 1 & 0 & \cdots & 0 \\
0 & 0 & 1 & \cdots & 0 \\
\vdots & \vdots & \vdots & \ddots & 1 \\
0 & 0 & 0 & \cdots & 0
\end{array}\right]_{n \times n} \\
& B=\left[\begin{array}{c}
0 \\
0 \\
\vdots \\
b
\end{array}\right]_{n \times 1}, \\
& C=\left[\begin{array}{llll}
1 & 0 & \cdots & 0
\end{array}\right]_{1 \times n}, \\
& B_{\omega}=B_{f}=\left[\begin{array}{c}
0 \\
0 \\
\vdots \\
1
\end{array}\right]_{n \times 1} \text {, }
\end{aligned}
$$

and $(A, C)$ is an observable pair which is verifiable by $\operatorname{rank}\left[\begin{array}{c}C \\ C A \\ \vdots \\ C A^{n-1}\end{array}\right]_{n \times n}=n$.

Based on (17), an observer-based residual generator can be designed as

$$
\begin{aligned}
& \dot{\hat{x}}=A \widehat{x}(t)+B u_{0}(t)+L(y(t)-\widehat{y}(t)), \\
& \widehat{y}=C \widehat{x}(t),
\end{aligned}
$$

where $L$ is the gain matrix which needs to be set.

Defining the state estimation and the residual, $e(t)=$ $x(t)-\widehat{x}(t)$ and $\varepsilon(t)=y(t)-\widehat{y}(t)$, respectively, the dynamics of the residual generator are governed by

$$
\begin{aligned}
\dot{e}(t) & =(A-L C) e(t)+B_{\omega} \omega(t)+B_{f} f(t), \\
\varepsilon & (t)=C e(t) .
\end{aligned}
$$

Denote the sum of fault and error by $f_{\emptyset}(t)=f(t)+$ $\omega(t)$. Note that $f_{\emptyset}(t)$ is input of the residual generator, so the relation between residual $\varepsilon(t)$ and input $f_{\emptyset}(t)$ can be definitely obtained to perform the task of fault diagnosis. In addition, the design and analysis in this paper are based on the assumptions as follows:

(A1) The real time action $\|\phi\|$ is bounded.
(A2) $b$ is a known constant.

(A3) $\omega(t)$ remains bounded before and after the occurrence of a fault; that is, $\|\omega(t)\| \leq \lambda_{\omega}$.

With this method, the fault diagnosis problem can be transformed into the problem of pole placement. Now the object is to compute the gain $L$ to make residual generator asymptotically stable and fulfill the following performance indicators:

(A1) Residual generator (20) is asymptotically stable when no fault occurs.

(A2) For the required property $\tau>0$, transfer function $G(s)$ between the sum $f_{\varrho}(t)$ and residual signal $\varepsilon(t)$ satisfies inequality $\|G(s)\|>\tau$ when a fault occurs.

Theorem 2. Consider the dynamics of residual generator (20) rewritten by

$$
\begin{aligned}
\dot{e}(t) & =(A-L C) e(t)+B_{\omega} \omega(t)+B_{f} f(t), \\
\varepsilon & (t)=C e(t) ;
\end{aligned}
$$

under the assumption given above, suppose the expected eigenvalues of filter (19) are $\lambda_{i}^{*}(i=1,2, \ldots, n)$. If there exist two symmetric positive definite matrices $P, Q$ and a state feedback matrix $\bar{K}$ verifying Lyapunov equation $(A-L C)^{T} P+$ $P(A-L C)=-Q$ and $\lambda_{i}\left(A-C^{T} \bar{K}\right)=\lambda_{i}^{*}$, respectively, then residual generator (19) is asymptotically stable, and also $L=\bar{K}^{T}$.

Proof. For the symmetric positive definite matrices $P, Q$, let us consider a quadratic Lyapunov function candidate

$$
V(t)=e^{T} P e .
$$

If there are no fault and uncertainty, that is, $f(t)=0$ and $\omega(t)=0$, we can get $\dot{V}(t)$ based on (20)

$$
\begin{aligned}
\dot{V}(t) & =\dot{e}^{T} P e+e^{T} P \dot{e} \\
& =e^{T}(A-L C)^{T} P e+e^{T} P(A-L C) e \\
& =e^{T}\left[(A-L C)^{T} P+P(A-L C)\right] e=-e^{T} Q e .
\end{aligned}
$$

Then we get $\dot{V}(t)<0$. Since $V(t) \rightarrow \infty$ as $\|e(t)\| \rightarrow \infty$, the residual generator (19) is globally asymptotically stable based on Lyapunov theorem.

As previously stated, $(A, C)$ is an observable pair. Thus, integrator series control system (16) is completely observable. And all the eigenvalues of residual generator (19) can be configured arbitrarily based on pole placement theorem; that is, there exists a state feedback matrix $\bar{K}$ such that $\lambda_{i}(A-$ $\left.C^{T} \bar{K}\right)=\lambda_{i}^{*}$.

Based on duality principle, the observability of $(A, C)$ is equivalent to the controllability of $\left(A^{T}, C^{T}\right)$. Computing dual coefficient matrices $\bar{A}=A^{T}, \bar{B}=C^{T}$, pole assignment method is employed to calculate state feedback matrix $\bar{K}$ with $(\bar{A}, \bar{B})$ and the expected eigenvalues $\lambda_{i}^{*}(i=1,2, \ldots, n)$. 
Therefore, the gain matrix is determined according to the conditions of convergence of the monitor and pole assignment method; that is, $L=\bar{K}^{T}$.

Remark 3. Let us denote the eigenvalues of the matrix $A-$ $C^{T} \bar{K}$ by $\lambda_{i}\left(A-C^{T} \bar{K}\right)$. The equality $\lambda_{i}\left(A-C^{T} \bar{K}\right)=\lambda_{i}^{*}$ means that all the eigenvalues of filter (19) can be configured arbitrarily.

From the dynamics of the residual generator (20), we can transfer function $G(s)$ between the sum $f_{\emptyset}(t)$ and residual signal $\varepsilon(t)$ as follows:

$$
\begin{aligned}
G(s) & =\frac{\varepsilon(s)}{F_{\emptyset}(s)}=C(s I-A+L C)^{-1} B_{f} \\
& =\frac{E_{n-1} s^{n-1}+E_{n-2} s^{n-2}+\cdots+E_{0}}{\alpha(s)},
\end{aligned}
$$

where

$$
\begin{aligned}
& \alpha(s)=\operatorname{det}(s I-A+L C)=s^{n}+\alpha_{n-1} s^{n-1}+\cdots+\alpha_{1} s \\
& +\alpha_{0} \\
& E_{n-1}=C B_{f} \\
& E_{n-2}=C A B_{f}+\alpha_{n-1} C B_{f} \text {, } \\
& E_{1}=C A^{n-2} B_{f}+\alpha_{n-1} C A^{n-3} B_{f}+\cdots+\alpha_{2} C B_{f} \\
& E_{0}=C A^{n-1} B_{f}+\alpha_{n-1} C A^{n-2} B_{f}+\cdots+\alpha_{1} C B_{f} .
\end{aligned}
$$

Then we have

$$
\begin{aligned}
F_{\oplus}(s) & =\frac{\varepsilon(s)}{C(s I-A+L C)^{-1} B_{f}} \\
& =\frac{\varepsilon(s) \cdot \alpha(s)}{E_{n-1} s^{n-1}+E_{n-2} s^{n-2}+\cdots+E_{0}} .
\end{aligned}
$$

By applying the inverse Laplace transformation to (26), we have

$$
\begin{aligned}
f_{\emptyset}(t) & =L^{-1}\left[\frac{\varepsilon(s)}{C(s I-A+L C)^{-1} B_{f}}\right] \\
& =L^{-1}\left[\frac{\varepsilon(s) \cdot \alpha(s)}{E_{n-1} s^{n-1}+E_{n-2} s^{n-2}+\cdots+E_{0}}\right] .
\end{aligned}
$$

The above equation shows the definite relationship between the sum $f_{\varrho}(t)$ and residual signal $\varepsilon(t)$. Also Theorem 2 indicates that if there is no fault, filter (19) can accurately track the state and output of system (17). That is to say, the residual signal is asymptotically stable at zero. When a fault occurs, there is a sudden change in the state and output of system (17), but filter (19) cannot track them timely. Thus, the residual signal is large at first which can be used to detect fault. From (27), we can approximately estimate the fault $f(t)$ based residual signal $\varepsilon(t)$ to identify fault. Now the comprehensive analysis proves that the proposed scheme is effective for fault diagnosis of system (1).

In addition, linearization of dynamic compensation ensures that the uncertainties would not interfere with fault diagnosis, because the uncertainties are offset by its estimation. References $[23,24]$ estimated fault directly using ESO to get its approximate function. Without doubt, this method took uncertainty as part of the fault in uncertain systems, which led to large error of estimated fault and actual fault such that the result of fault diagnosis is inaccurate. Compared with $[23,24]$, the method in this paper is complex to some extent. But the proposed fault diagnosis method based on linear system is mature and applicable. The most important thing is that the proposed method is more robust.

\section{Threshold Analysis}

Robust technique is employed in this paper to enhance the reliability of fault detection and reduce the false alarm rate. There are two kinds of robust techniques: the unknown input decoupled observer technique and the limited threshold technique. For uncertainties of the system, threshold treatment technique is adopted to realize robust fault evaluation in the algorithm. When the residual signal exceeds the defined threshold, we can identify that there exists fault based on the mechanism. Otherwise, there is no fault.

Theorem 4. For system (4), ESO (6) is designed to estimate the states, and also the observer-based residual generator (19) is designed to diagnose fault. If there is no fault, the residual signal satisfies the following inequality for the time domain $t \in\left[t_{1}, t_{2}\right]$ :

$$
\|\varepsilon(t)\| \leq \lambda_{1} T \lambda_{\omega}
$$

Proof. By defining the state transfer matrix $\Theta(t)$, then we can get

$$
\Theta(t, \tau)=e^{(A-L C)(t-\tau)}
$$

For $t \in\left[t_{1}, t_{2}\right]$, we have

$$
\begin{aligned}
e(t)= & \Theta\left(t, t_{1}\right) e\left(t_{1}\right)+\int_{t_{1}}^{t} \Theta(t, \tau) B_{f} f(\tau) d_{\tau} \\
& +\int_{t_{1}}^{t} \Theta(t, \tau) B_{\omega} \omega(\tau) d_{\tau} .
\end{aligned}
$$

At the initial time $t_{1}$, state estimations are the same as true values; that is, $\widehat{x}\left(t_{1}\right)=x\left(t_{1}\right)$; that is, $e\left(t_{1}\right)=0$.

Based on norm theory, we obtain

$$
\begin{aligned}
\|e(t)\| \leq & \left\|\int_{t_{1}}^{t} \Theta(t, \tau) B_{f} f(\tau) d_{\tau}\right\| \\
& +\left\|\int_{t_{1}}^{t} \Theta(t, \tau) B_{\omega} \omega(\tau) d_{\tau}\right\|
\end{aligned}
$$




$$
\begin{aligned}
\leq & \int_{t_{1}}^{t}\left\|\Theta(t, \tau) B_{f} f(\tau)\right\| d_{\tau} \\
& +\int_{t_{1}}^{t}\left\|\Theta(t, \tau) B_{\omega} \omega(\tau)\right\| d_{\tau} .
\end{aligned}
$$

By substituting the above inequality into (20), then we have

$$
\begin{aligned}
\|\varepsilon(t)\|= & \|C e(t)\| \\
\leq & \int_{t_{1}}^{t}\left\|C \Theta(t, \tau) B_{f} f(\tau)\right\| d_{\tau} \\
& +\int_{t_{1}}^{t}\left\|C \Theta(t, \tau) B_{\omega} \omega(\tau)\right\| d_{\tau} \\
= & \int_{t_{1}}^{t}\left\|C \Theta(t, \tau) B_{\omega} \omega(\tau)\right\| d_{\tau} \leq \lambda_{1} T \lambda_{\omega},
\end{aligned}
$$

where $T$ is the length of the time domain; that is, $T=t_{2}-t_{1}$. And

$$
\lambda_{1}=\sup _{t, \tau \in\left[t_{1}, t_{2}\right]}\left\|C \Theta(t, \tau) B_{\omega}\right\| .
$$

Therefore the threshold $\Gamma$ can be set as $\lambda_{1} T \lambda_{\omega}$; that is, $\Gamma=$ $\lambda_{1} T \lambda_{\omega}$. Then the fault detection can be performed using the following mechanism:

$$
\begin{aligned}
& \|\varepsilon(t)\| \leq \Gamma, \text { there is no fault, } \\
& \|\varepsilon(t)\|>\Gamma, \text { there is a fault. }
\end{aligned}
$$

\section{Example and Simulation}

Consider the following nonlinear system with a fault:

$$
\begin{aligned}
\dot{x}_{1}= & x_{2}(t), \\
\dot{x}_{2}= & \cos 0.6 t \cdot x_{1}(t)+\cos 0.7 t \cdot x_{2}(t) \\
& +0.5 \operatorname{sign}(\sin t)+u(t)+\omega(t)+f(t), \\
y= & x_{1} .
\end{aligned}
$$

From (35), we can know that nonlinear function is

$$
\begin{aligned}
\phi\left(x_{1}, x_{2}, t\right)= & \cos 0.6 t \cdot x_{1}(t)+\cos 0.7 t \cdot x_{2}(t) \\
& +0.5 \operatorname{sign}(\sin t) .
\end{aligned}
$$

The combination of uncertainty, fault, and nonlinear function $\Phi\left(x_{1}, x_{2}, t\right)=\phi\left(x_{1}, x_{2}, t\right)+\omega(t)+f(t)$ is tracked by ESO which can be designed based on system (6). And so are the states $x_{1}, x_{2}$. According to the estimations of states and system structure, the estimation of nonlinear function $\widehat{\phi}\left(\widehat{x}_{1}, \widehat{x}_{2}, t\right)$ can be obtained.

In the simulation example, $\omega(t)$ is white noise with an energy of 0.01 , permissible estimating error $\gamma=0.8$, timedomain length $T=5$, and the fault detection threshold $\Gamma=5$.
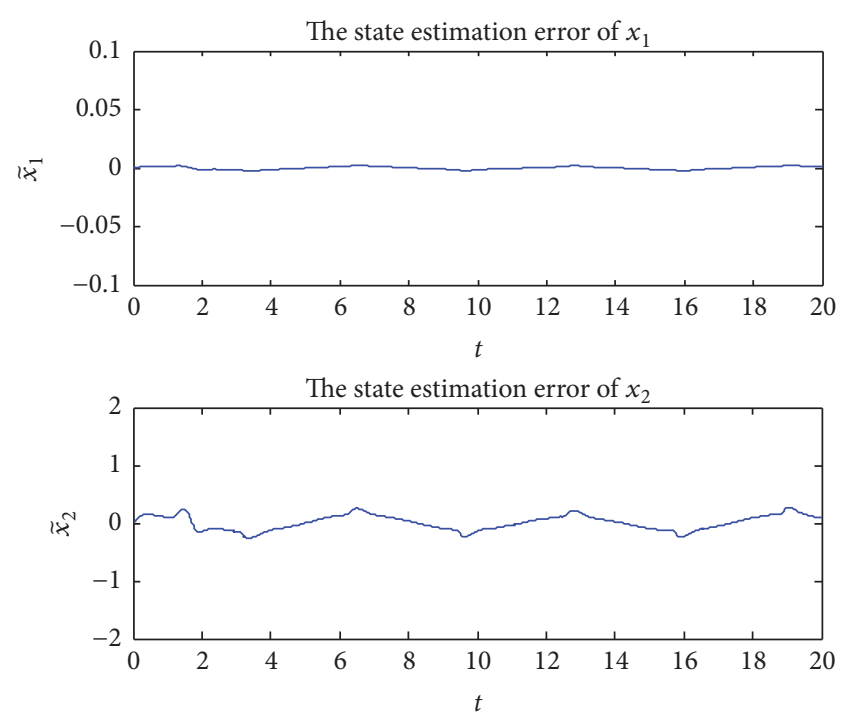

FIGURE 1: State estimation errors for $f_{1}(t)$.

The parameters of ESO are adopted as $a=0.01, \delta=0.01, \beta_{1}=$ $100, \beta_{2}=65, \beta_{3}=80$, and step length $h=0.01$. And the faulty signal is nominated as having two types:

$$
\begin{aligned}
& f_{1}(t)= \begin{cases}0, & t<0, \\
3.5 \sin t, & t \geq 0,\end{cases} \\
& f_{2}(t)= \begin{cases}0, & t<0, \\
3.5 t, & t \geq 0 .\end{cases}
\end{aligned}
$$

The simulation considers the effect of the two fault signals separately. When sine function fault $f_{1}(t)$ occurs, the state estimation errors are given in Figures 1 and 2 showing the combination of uncertainty, fault, and nonlinear function $\Phi\left(x_{1}, x_{2}, t\right)=\phi\left(x_{1}, x_{2}, t\right)+\omega(t)+f(t)$ tracking trajectory and it can be seen from this figure that a good tracking performance of the proposed ESO is achieved. Figure 3 shows the capability of the proposed method to track sine function fault $f_{1}(t)$ and diagnose the fault. When ramp function fault $f_{2}(t)$ occurs, the state estimation errors given by ESO are illustrated in Figure 4, and the tracking effects of ESO on the combination $\Phi\left(x_{1}, x_{2}, t\right)$ can be seen in Figure 5. Figure 6 shows the ramp function fault signal $f_{2}(t)$ and its estimation. From Figures 1 and 4 and Figures 2 and 5, it is seen that all the true values are approached by the estimated ones fleetly on the system states and the combination which is estimated by the ESO. The existence of band-limited white noise influences the accuracy of estimation, but the fault estimations can also track them timely which we can know from Figures 3 and 6.

On the other hand, it can be shown from Figures 1-6 that system states $x_{1}, x_{2}$ themselves have sharply periodic oscillation and their oscillatory period is $6 \mathrm{~s}$. Although the oscillation has a great effect on fault diagnosis of the system and tracking performance, the tracking errors are converged and the fault estimations are accurate. That is, the variation of tracking error is caused by the periodic oscillation of states 


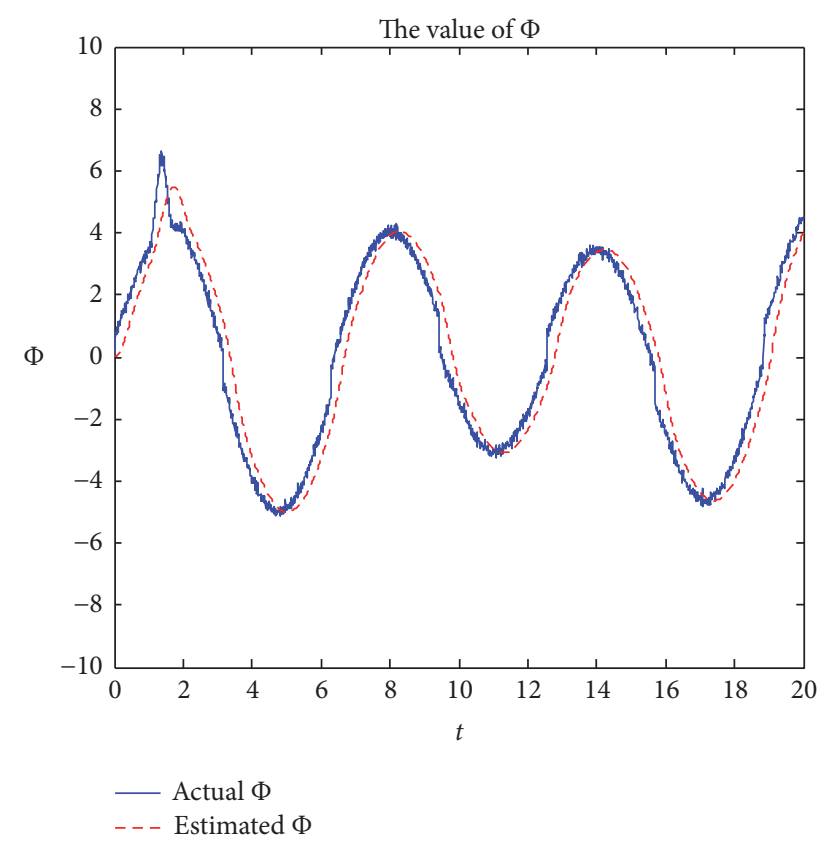

Figure 2: Combination $\Phi\left(x_{1}, x_{2}, t\right)$ estimate and true value for $f_{1}(t)$.

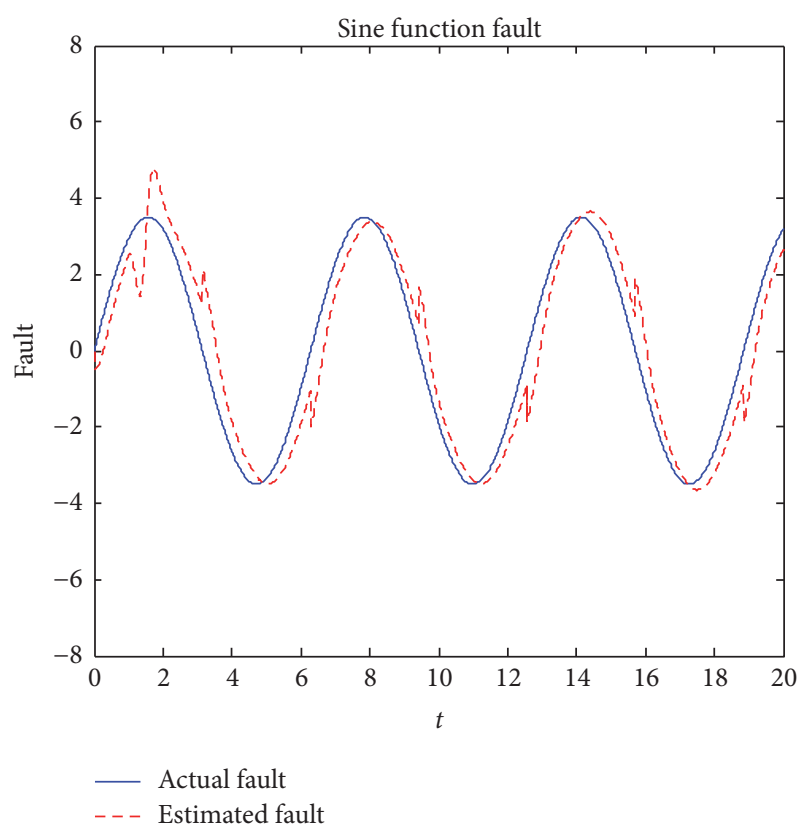

FIGURE 3: $f_{1}(t)$ and its estimation.

$x_{1}, x_{2}$. Therefore, the proposed method needs to overcome the oscillation to track states and faults. The simulation results illustrate that the tracking of states and faults can be achieved by the proposed method.

\section{Conclusion}

In this paper, based on the ESO of the active disturbance rejection controller and linearization of dynamic compensation, a robust fault diagnosis scheme for a class of nonlinear uncertain systems with triangular form is presented.
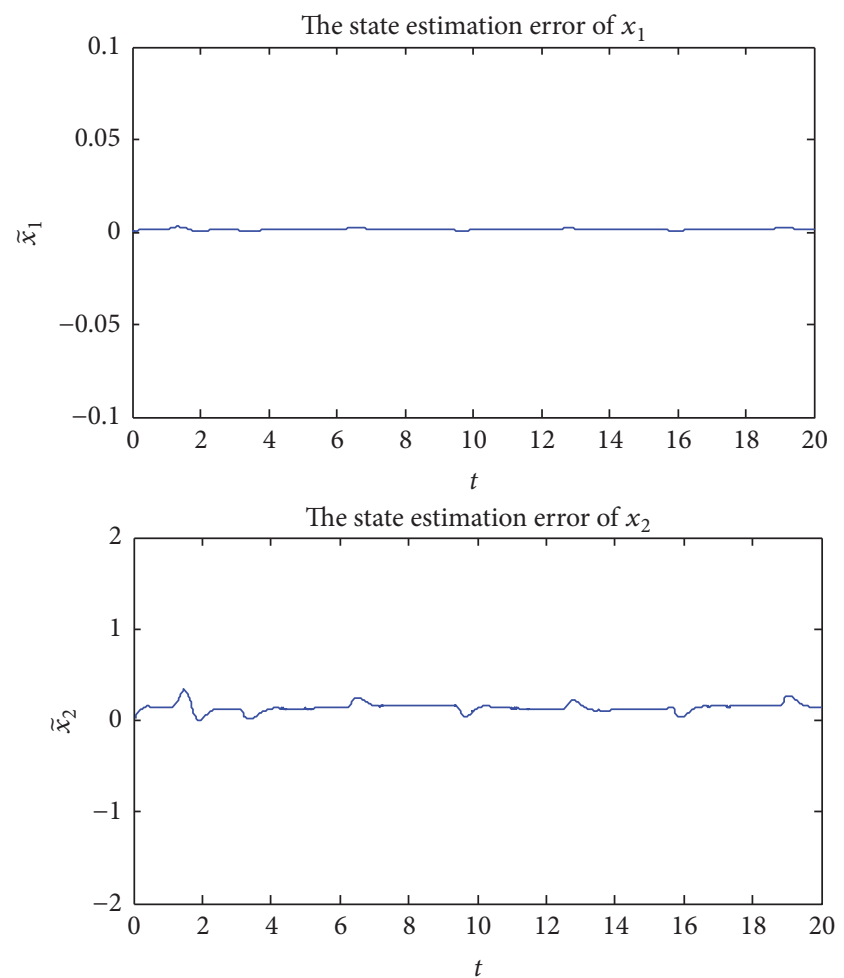

FIGURE 4: State estimation errors for $f_{2}(t)$.

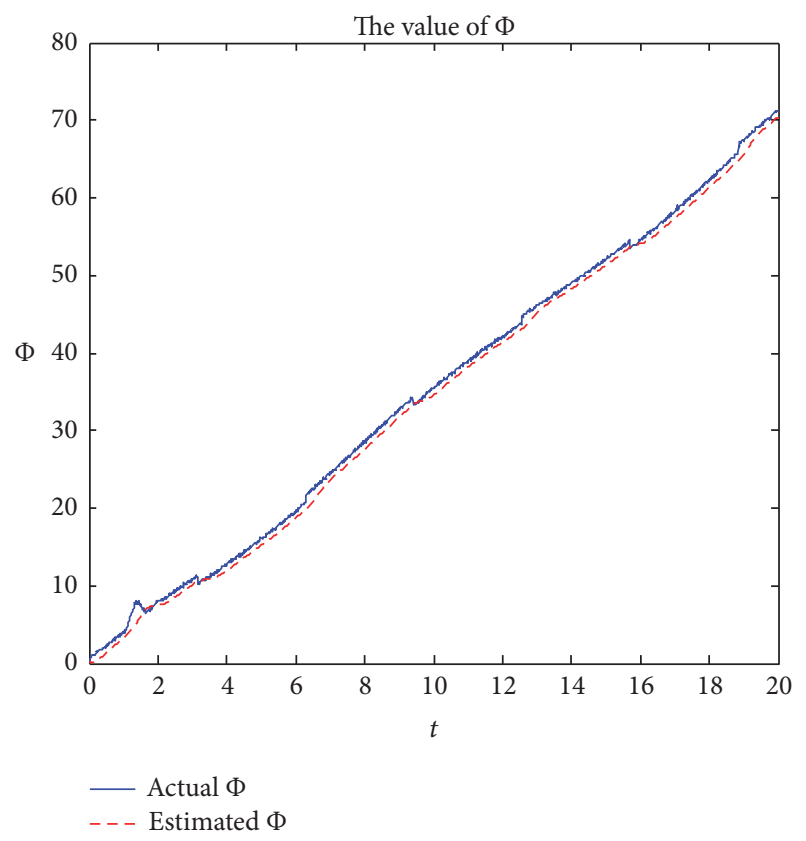

FIgURE 5: Combination $\Phi\left(x_{1}, x_{2}, t\right)$ estimate and true value for $f_{2}(t)$.

Compared with other methods, a good advantage is that the proposed method is more robust. The combination of uncertainty, fault, and nonlinear function is defined as a new system state. The ESO has a good tracking performance for system states and the combination. Then nonlinear system is linearized with state estimations by linearization of dynamic 


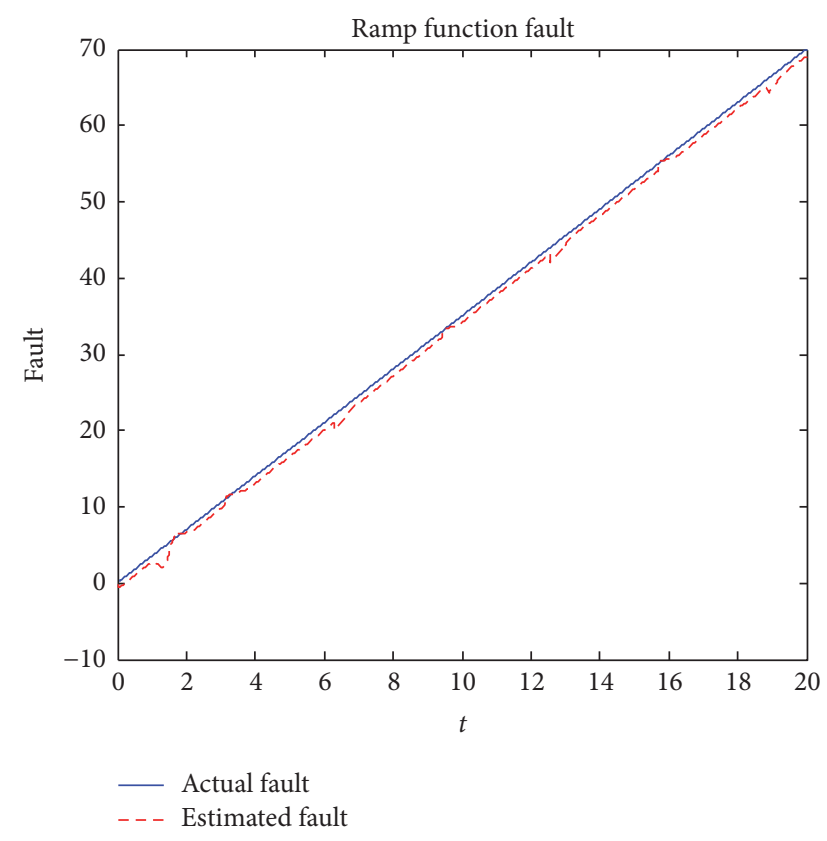

Figure 6: $f_{2}(t)$ and its estimation.

compensation to realize fault diagnosis. The robustness and fault detection are enhanced via the appropriately designed threshold in the diagnostic decision-making stage. A simulation example is used to illustrate the effectiveness of the proposed method.

\section{Conflicts of Interest}

The authors declare that they have no conflicts of interest.

\section{Acknowledgments}

This work is supported by National Natural Science Foundation (NNSF) of China under Grant no. 61305117, 973 subproject, and National Key Laboratory Foundation of China under Grant 9140c59030411ht05.

\section{References}

[1] P. M. Frank, "Fault diagnosis in dynamic systems using analytical and knowledge-based redundancy: a survey and some new results," Automatica, vol. 26, no. 3, pp. 459-474, 1990.

[2] J. Chen and R. J. Patton, "Fault diagnosis of non-linear dynamic systems," in Robust Model-Based Fault Diagnosis for Dynamic Systems, vol. 3 of The International Series on Asian Studies in Computer and Information Science, pp. 251-295, Springer, Boston, MA, USA, 1999.

[3] V. Venkatasubramanian, R. Rengaswamy, K. Yin, and S. N. Kavuri, "A review of process fault detection and diagnosis part I: quantitative model-based methods," Computers and Chemical Engineering, vol. 27, no. 3, pp. 293-311, 2003.

[4] X. Zhang, M. M. Polycarpou, and T. Parisini, "Fault diagnosis of a class of nonlinear uncertain systems with Lipschitz nonlinearities using adaptive estimation," Automatica. A Journal of IFAC, the International Federation of Automatic Control, vol. 46, no. 2, pp. 290-299, 2010.

[5] J. S. Tsai, M.-H. Lin, C.-H. Zheng, S.-M. Guo, and L.-S. Shieh, "Actuator fault detection and performance recovery with Kalman filter-based adaptive observer," International Journal of General Systems, vol. 36, no. 4, pp. 375-398, 2007.

[6] J. Guo, X. Huang, and Y. Cui, "Design and analysis of robust fault detection filter using LMI tools," Computers and Mathematics with Applications, vol. 57, no. 11-12, pp. 1743-1747, 2009.

[7] H. R. Karimi, M. Zapateiro, and N. Luo, "A linear matrix inequality approach to robust fault detection filter design of linear systems with mixed time-varying delays and nonlinear perturbations," Journal of the Franklin Institute. Engineering and Applied Mathematics, vol. 347, no. 6, pp. 957-973, 2010.

[8] S. X. Ding, "Fault Isolation Schemes," in Model-Based Fault Diagnosis Techniques, Advances in Industrial Control, pp. 405440, Springer, 2013.

[9] T. Mulumba, A. Afshari, K. Yan, W. Shen, and L. K. Norford, "Robust model-based fault diagnosis for air handling units," Energy and Buildings, vol. 86, pp. 698-707, 2015.

[10] L. Li and D. Zhou, "Fast and robust fault diagnosis for a class of nonlinear systems: Detectability analysis," Computers and Chemical Engineering, vol. 28, no. 12, pp. 2635-2646, 2004.

[11] F. Bagheri, H. Khaloozaded, and K. Abbaszadeh, "Stator fault detection in induction machines by parameter estimation, using adaptive Kalman filter," in Proceedings of the 2007 Mediterranean Conference on Control and Automation, MED, July 2007.

[12] P. Seiler, J. Bokor, B. Vanek, and G. J. Balas, "Robust model matching for geometric fault detection filters," in Proceedings of the 2011 American Control Conference, ACC 2011, pp. 226-231, San Francisco, CA, USA, July 2011.

[13] W. Wang and C. Wen, "Adaptive actuator failure compensation control of uncertain nonlinear systems with guaranteed transient performance," Automatica. A Journal of IFAC, the International Federation of Automatic Control, vol. 46, no. 12, pp. 2082-2091, 2010.

[14] W. Wang and C. Wen, "Adaptive compensation for infinite number of actuator failures or faults," Automatica. A Journal of IFAC, the International Federation of Automatic Control, vol. 47, no. 10, pp. 2197-2210, 2011.

[15] R. Sun, S. Liu, and Y.-F. Zhang, "Design of fault diagnosis observer for a class of nonlinear systems," Kongzhi Lilun Yu Yingyong/Control Theory and Applications, vol. 30, no. 11, pp. 1462-1466, 2013.

[16] R. Patton J, J. Chen, and B. Nielsen S, "Model-based methods for fault diagnosis: some guide-lines," Transactions of the Institute of Measurement Control, vol. 17, no. 2, pp. 73-83, 1995.

[17] X. Wu and B. Zhou, "Fault tolerance control for proton exchange membrane fuel cell systems," Journal of Power Sources, vol. 324, pp. 804-829, 2016.

[18] J. Huang, Z. Jiang, and T. Xu L, "Method on component fault diagnosis for nonlinear systems," Control and Decision, vol. 29, no. 5, pp. 925-928, 2014.

[19] X. Qing-shi and G. Ying-qing, "Fault diagnosis of aircraft engines based on adaptive sliding mode observers," Aeronautical Computing Technique, 2016.

[20] J. Guo W, L. Gou F, and P. Shi Y, "Research on Fault Detection Method Based on Adaptive Observer," Computer Simulation, vol. 31, no. 2, pp. 136-130, 2014.

[21] G. Stefatos and A. Ben Hamza, "Dynamic independent component analysis approach for fault detection and diagnosis," Expert Systems with Applications, vol. 37, no. 12, pp. 8606-8617, 2010. 
[22] D. Ye, P. Lin, and Z. Gao, "Research on fault diagnosis using extended state observer," Chinese Journal of Scientific Instrument, 2008.

[23] K. Chang and WU. Guo-Qing, "Fault diagnosis method for nonlinear systems via ESO," Journal of Hebei University of Science, 2012.

[24] K. Chang and G. Q. Wu, "Application of ESO in fault detection and identification for the nonlinear systems," Information Technology, 2013.

[25] Y. Yan B, Research on several methods of fault diagnosis for nonlinear systems and their applications, Shanghai Jiao Tong University, 2010.

[26] J. Chu, J. Yu, and J. C. Wang, "Design of nonlinear robust controller based on state transformation," Acta Automatica Sinica, vol. 18, no. 5, pp. 590-594, 1992. 


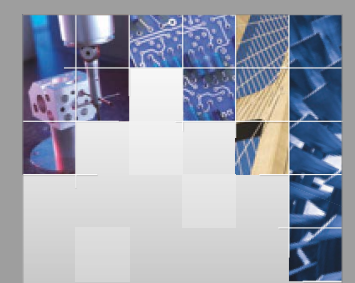

\section{Enfincering}
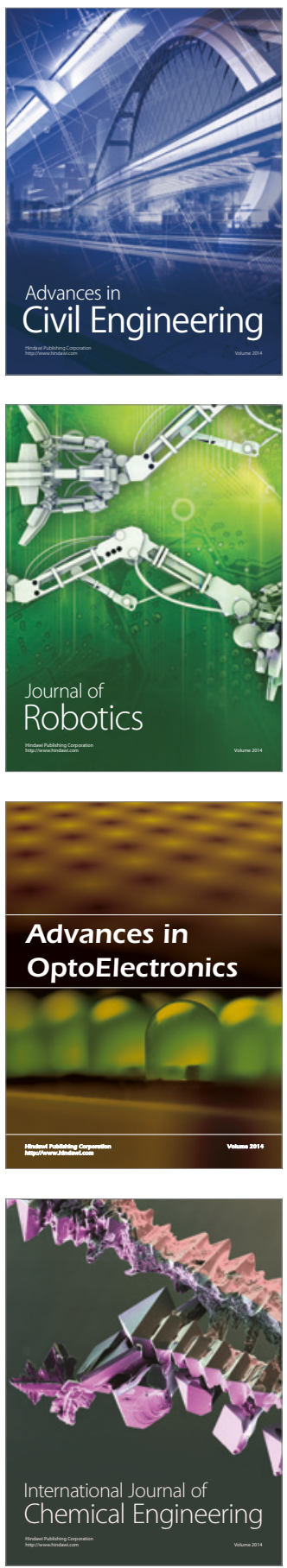

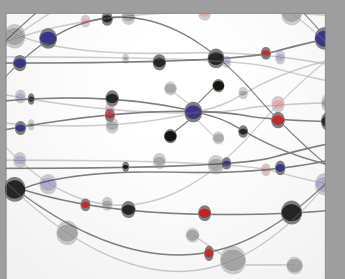

The Scientific World Journal

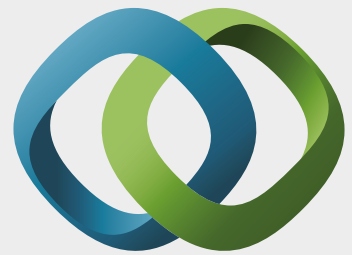

\section{Hindawi}

Submit your manuscripts at

https://www.hindawi.com
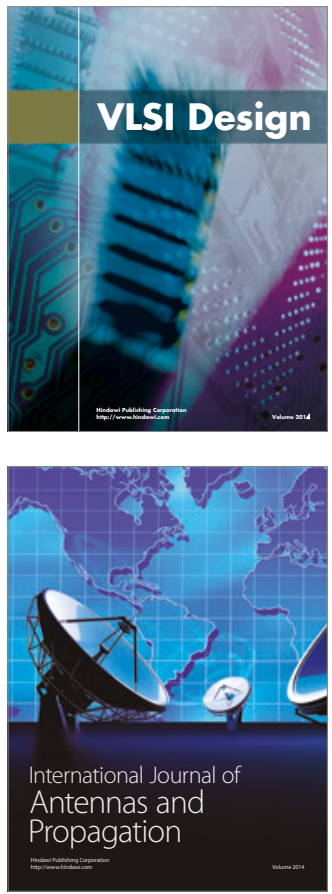

\section{Rotating}

Machinery
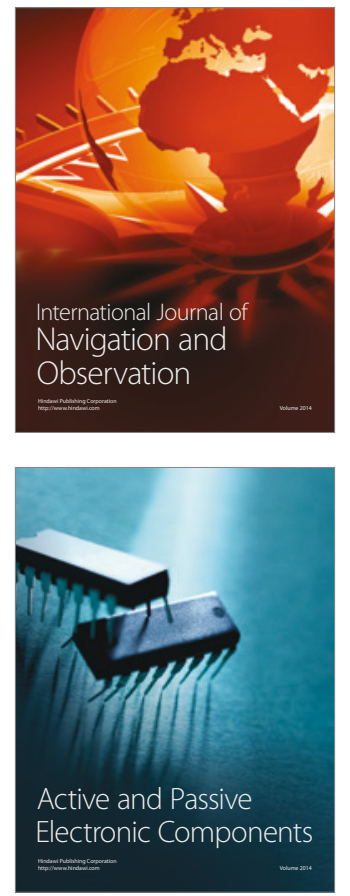
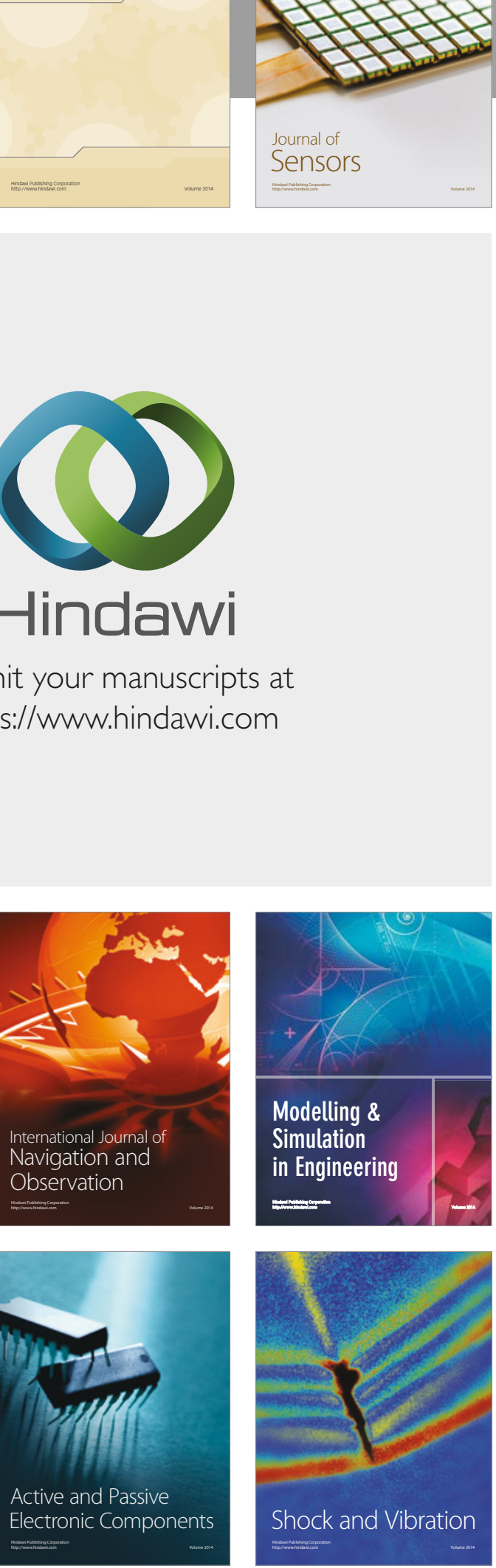
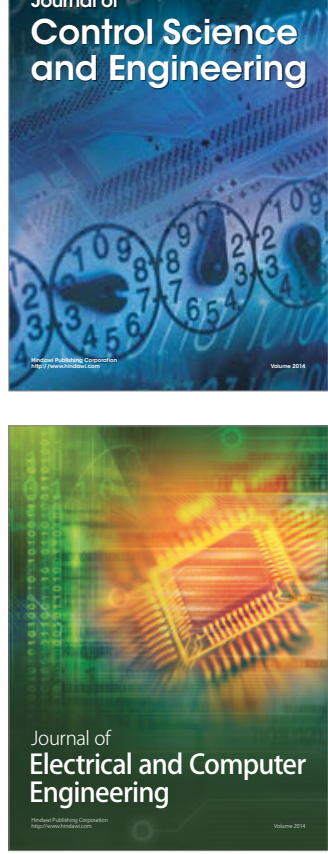

Distributed

Journal of

Control Science

and Engineering
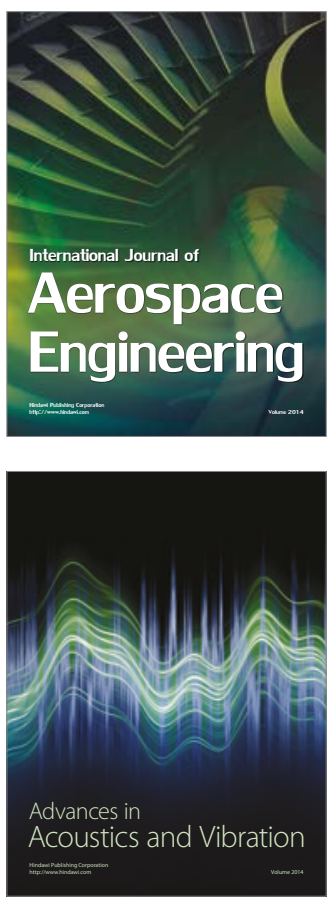

Sensor Networks 